\title{
A bronchogenic cyst with atrial flutter
}

\author{
Erhan Tenekecioglu', Mustafa Yılmaz' \\ ${ }^{1}$ Department of Cardiology, Bursa Yuksek Ihtisas Education and Resarch Hospital, Bursa, Turkey
}

\section{A B S TR A C T}

The majority of bronchogenic cysts occur in the mediastinum and especially within the pulmonary parenchyma. Bronchogenic cysts have various clinical and radiological manifestations. In adults, these are often asymptomatic and most of them are only incidentally recognized in imaging procedures. In infancy and early childhood, compression of the tracheobronchial tree leads to symptoms and occasionaly life-threatening complications. Bronchogenic cysts have been stated rarely among the causes of an arythmia in early childhood. We reported a 7-years old female presented with paroxysmal palpitation continuing for one month. Contrast enhanced computed tomography showed cystic mass in the right hemi-thorax, compressing the right atrium. After surgical removal histopathologic examination revealed a lining of respiratory epithelium associated with a wall containing smooth muscle, a finding that is characteristic of bronchogenic cyst.

Key words: Bronchogenic cyst, Atrial flutter, Cystic mass, Mediastinal cyst, Contrast enhanced computed tomography

\section{INTRODUCTION}

Bronchogenic cysts are congenital anomalies of the ventral foregut. The majority occur in the mediastinum and especially within the pulmonary parenchyma. Bronchogenic cysts are the most common of mediastinal cysts and have various clinical and radiological manifestations. ${ }^{1}$ In adults, these are often asymptomatic and are only incidentally recognized in imaging procedures. Chest pain, discomfort, cough and dyspnea are common symptoms. ${ }^{2,3}$ In infancy and early childhood, compression of the tracheobronchial tree leads to symptoms and occasionaly life-threatening complications. Bronchogenic cysts have been stated rarely among the causes of an arythmia in early childhood. Contrast enhanced computed tomography showed a giant cystic mass with a maximum diameter of $6 \mathrm{~cm}$ located in the right hemi-thorax, compressing the right atrium.

\section{CASE REPORT}

A 7-years old girl presented with paroxysmal palpitation continuing for one month. In the physical examination tachycardia was detected; among the vital signs the blood pressure was $120 / 80 \mathrm{mmHg}$; and axillary temperature was $36.5^{\circ} \mathrm{C}$. On admission, the electrocardiogram (ECG) showed atrial flutter with variable atrioventricular conduction. Chest radiography revealed cardiomegaly that right ventricle and atrium appears as dilated (Figure 1). Two-dimensional echocardiography demonstrated the presence of an extracardiac mass that compressing the right atrium laterally. There was no other cardiac or valvular abnormality during the transthoracic echocardiography. Contrast enhanced computed tomography showed a giant cystic mass with a maximum diameter of $6 \mathrm{~cm}$ located in the right hemi-thorax, compressing the right atrium (Figure 2). Since hemodynamic destabilization, the patient was treated with intravenous digitalization. Frequent episodes of paroxysmal atrial flutter with slower ventricular rates persisted, alternating with brief periods of sinus rhythm. Based on these findings and the general consensus that surgery has been advocated as the treatment of choice for symptomatic bronchogenic cyst, the patient was admitted for elective removal of the mediastinal mass. Morphologically, the cystic content was of a liquid nature. Histopathologic examination of the lesion showed a lining of respiratory epithelium associated with a wall containing smooth muscle, a finding that is characteristic of 
bronchogenic cyst (Figure 3). The patient's postoperative course was uneventful and she left the hospital on the 8th postoperative day.

\section{DISCUSSION}

Bronchogenic cysts may occur in any part of the thoracic cavity. Majority are located near the tracheal carina in the middle or posterior mediastinum. They may occur within the lung parenchyma, pleura, or diaphragm. ${ }^{4}$ These

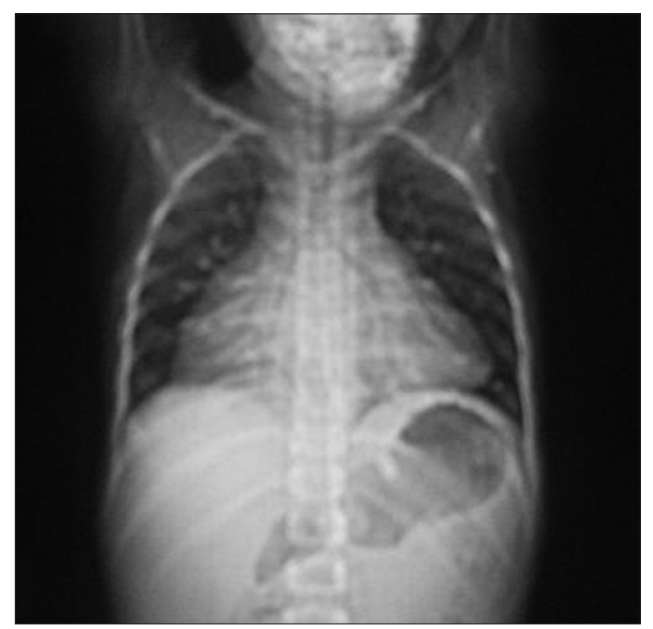

Figure 1: Chest radiography revealed cardiomegaly that right ventricle and atrium appears as dilated

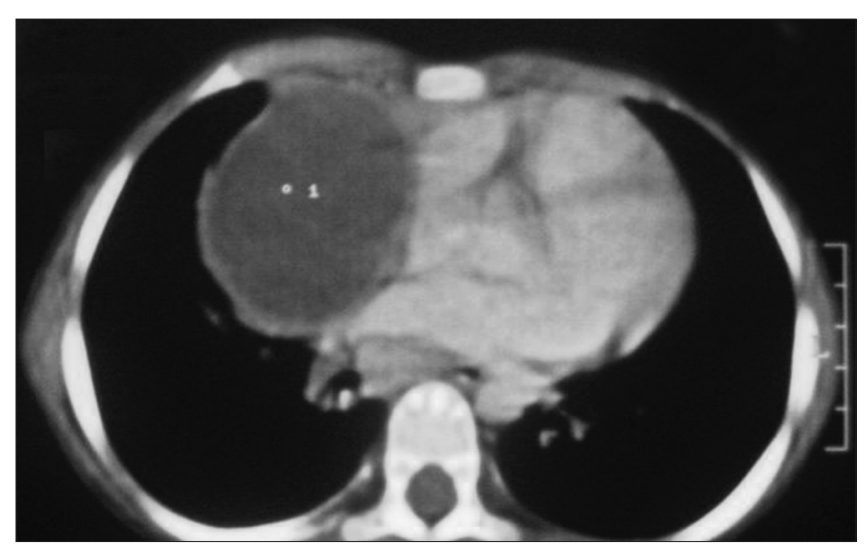

Figure 2: Contrast enhanced computed tomography showed a giant cystic mass located in middle mediastinum, compressing the right atrium

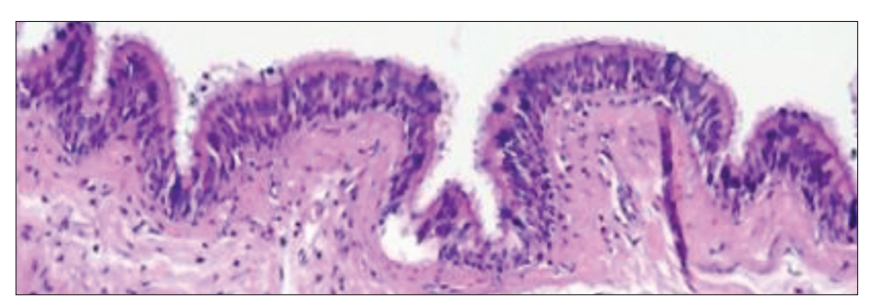

Figure 3: The bronchogenic cyst is lined by bronchiolar epithelium and thick fascicles of smooth muscle in the wall congenital masses are occasionally found in association with other congenital pulmonary malformations such as sequestrations. ${ }^{5}$ Bronchogenic cysts may undergo an abrupt increase in size as a result of hemorrhage or infection. Most are asymptomatic, but they may occasionally cause symptoms secondary to compression of adjacent structures. On chest radiographs, a bronchogenic cyst usually appears as a well-defined solitary mass with homogeneous opacity just inferior to the carina and often protruding slightly toward the right hilar shadow. Imaging techniques such as computed tomography and 2-dimensional echocardiography are usefull for diagnosing those extracardiac masses.

Cardiovascular manifestations of bronchogenic cysts have rarely been reported. To our knowledge, arythmia associated with bronchogenic cyst in early childhood is uncommon in the literature. In the reports bronchogenic cysts caused tachyarrhythmia and the mechanism was compression and irritation to the heart mechanically. ${ }^{6}$ Previously, atrial fibrillation and atrial flutter has been reported in children with bronchogenic cysts for clinical manifestations of these cysts. ${ }^{6-8}$

In early childhood atrial flutter is not common and should be investigated cautiously to identify the underlying pathology. Patients who have undergone surgical repair of atrial septal defect, total anomalous pulmonary venous connection, and tetralogy of Fallot repair may later develop atrial flutter.

Treatment of bronchogenic cysts in pediatric patients is surgical excision. Before occurrence of any complication or symptom, surgical treatment should be recommended to prevent progressive growth and compression of thoracic structures.

\section{CONCLUSION}

The present case highlights that atrial flutter in childhood should be investigated delicately. Atrial flutter is infrequent in children and in case of atrial flutter without any surgical history, congenital heart disease should be considered and bronchogenic cyst should be investigated for an underlying pathology.

\section{REFERENCES}

1. Shaker W, Meatchi T, Dusser D and Riquet M. An unusual presentation of solitary fibrous tumour of the pleura: right atrium and inferior vena cava compression. European Journal of Cardio-Thoracic Surgery 2002; 22: 640-642.

2. Sarper A, AytenA, Golbasi I, Demircan A and Isin E. Bronchogenic cyst. Tex Heart Inst J 2003; 30:105-108. 
3. Limaiem $\mathrm{F}$, Ayadi-Kaddour A, Djilani $\mathrm{H}$, Kilani $\mathrm{T}$ and $\mathrm{El}$ Mezni F. Pulmonary and mediastinal bronchogenic cysts: a clinicopathologic study of 33 cases. Lung 2008; 186:55-61.

4. McAdams HP, Kirejczyk WM, Rosado-de-Christenson ML and Matsumoto S. Bronchogenic cyst: imaging features with clinical and histopathologic correlation. Radiology 2000; 217:441-446.

5. Groskin SA. Embryology of the lung and pulmonary abnormalities of developmental origin. In: Groskin SA, eds. Heitzman's the lung: radiologic-pathologic correlations. 3rd ed. St Louis, Mo: Mosby, 1993; pp. 13-42.

6. Fujino S, Hwang EH, Sekido N, Kaizaki Y, Arai $Y$ and Aoyama T. Paroxysmal atrial fibrillation due to bronchogenic cyst. Intern
Med. 2010; 49(19): 2107-2111.

7. Volpi A, Cavalli A, Maggioni AP and Pieri-Nerli F. Left atrial compression by a mediastinal bronchogenic cyst presenting with paroxysmal atrial fibrillation. Thorax 1988; 43:216-217.

8. Sanchez-Recalde A, Peinado Peinado R, Elvira González A and Antonio Sobrino J. Atrial flutter resulting from right atrial compression by a hepatic hydatid cyst. International Journal of Cardiology 2000; 73:87-89.

9. Biviano A, Garan H, Hickey K, Whang W, Dizon J and Rosenbaum M. Atrial flutter catheter ablation in adult patients with repaired tetralogy of Fallot: mechanisms and outcomes of percutaneous catheter ablation in a consecutive series. J Interv Card Electrophysiol 2010;28(2):125-135.

\section{Authors Contribution: \\ All authors contributed equally towards publication}

Source of Support: Nil, Conflict of Interest: None declared. 